\title{
Delayed recovery from initial orthostatic hypotension: an expression of frailty in the elderly
}

\author{
Daan J. L. van Twist ${ }^{1}$ - Guy J. M. Mostard ${ }^{1}$ - Walther M. W. H. Sipers ${ }^{2}$
}

Received: 25 December 2019 / Accepted: 26 December 2019 / Published online: 28 January 2020

(c) Springer-Verlag GmbH Germany, part of Springer Nature 2020

Initial orthostatic hypotension $(\mathrm{OH})$ is a clinical syndrome with a transient drop in blood pressure upon standing (by definition $>40 \mathrm{mmHg}$ systolic or $>20 \mathrm{mmHg}$ diastolic within $15 \mathrm{sec}$ after standing up from supine position) [1]. This is different from classic $\mathrm{OH}$ which is defined by a sustained reduction of at least $20 \mathrm{mmHg}$ in systolic or $10 \mathrm{mmHg}$ in diastolic blood pressure within $3 \mathrm{~min}$ of standing [2]. The pathophysiological mechanisms leading to initial $\mathrm{OH}$ appear to differ from classic $\mathrm{OH}$, as initial $\mathrm{OH}$ typically occurs upon active standing and not during passive tilting. Therefore, the active use of leg and abdominal muscles appears to be involved. It has been hypothesized that muscle contraction increases venous return to the right atrium and slightly increases arterial blood pressure, especially in elderly $[1,3]$. The subsequent stimulation of mechanoreceptors in the right atrium and arterial baroreceptors results in abrupt withdrawal of sympathetic activity. Immediately after this occurs, muscle contraction leads to local arterial vasodilation during the first $4 \mathrm{~s}$ after contraction. Together, this results in a fall in systemic vascular resistance for 6-8 s after standing up. After the initial reduction, blood pressure usually recovers in about $20 \mathrm{~s}$. This recovery can be delayed in the elderly and in patients with neurodegenerative disorders.

In this issue of Clinical Autonomic Research, Saedon et al. report the results of the Malaysian Elders Longitudinal Research (MELoR) study [4]. They evaluated the association between postural changes in blood pressure and physical, functional, and cognitive performance in 1245 community-dwelling Asian elders (mean age

Daan J. L. van Twist

d.vantwist@zuyderland.nl

1 Department of Internal Medicine, Subdivision of Vascular Medicine, Zuyderland Medical Centre, PO Box 5500, 6130 MB Sittard/Heerlen, The Netherlands

2 Department of Geriatric Medicine, Zuyderland Medical Centre, Sittard/Heerlen, The Netherlands
67 years), with a relatively low baseline blood pressure (mean 113/69 mmHg). The authors found that 13\% of the participants had initial $\mathrm{OH}, 12 \%$ had classic $\mathrm{OH}$, and 50\% had a "combination" of both initial and classic $\mathrm{OH}$. The latter group is controversial since this combination is usually considered to be classic $\mathrm{OH}$ alone (and not a combination with initial $\mathrm{OH}$ ), as the drop in blood pressure in initial $\mathrm{OH}$ must, by definition, be transient [1]. However, these definitions might require updating, as previous studies demonstrated that many asymptomatic individuals fulfill the hemodynamic criteria for initial $\mathrm{OH}$ : in The Irish Longitudinal Study on Ageing (TILDA) for example, approximately $70 \%$ of the participants met the criteria for initial $\mathrm{OH}$ [5]. Moreover, the 95th percentile of initial systolic blood pressure drop was $59.6 \mathrm{mmHg}$ in men of 50 years old, substantially higher than the $40 \mathrm{mmHg}$ cutoff. Likewise, in the study by Saedon et al., 63\% of participants had an initial drop in blood pressure greater than the currently used cutoff values. Furthermore, in a cohort of patients evaluated for syncope, $50 \%$ met the criteria for initial $\mathrm{OH}$, but initial $\mathrm{OH}$ was considered to be the cause of syncope in only $~ 10 \%$ of the patients [6]. However, as most individuals in these studies were asymptomatic, it is questionable whether initial $\mathrm{OH}$ should be considered pathologic in all circumstances. Therefore, a redefinition of the cutoff values for initial $\mathrm{OH}$ might be necessary.

Perhaps a bit surprisingly, Saedon et al. found that participants with initial $\mathrm{OH}$ had better physical function, cognitive performance, and were better able to perform tasks in daily living as compared to participants with classic $\mathrm{OH}$. These findings persisted after adjustment for age, gender, diabetes mellitus, and baseline blood pressure. The authors concluded that initial $\mathrm{OH}$ is not a marker of frailty.

This, however, is presumably only one side of the coin. The other side of the coin is that individuals in whom the orthostatic blood pressure reductions take more time to recover to normal (referred to as delayed recovery) are in fact frailer than those in whom blood pressure recovers 
quickly (i.e., those with initial $\mathrm{OH}$ ). Of the tests used in this study, the timed "up-and-go" test provides a good estimate of the impact of $\mathrm{OH}$ in daily life: stand up from a chair, walk $3 \mathrm{~m}$ and sit back down again. This activity is directly influenced by orthostatic blood pressure changes and the ability to recover, in contrast to other parameters such as hand grip strength and cognitive performance. Saedon et al. showed that the time required to "up-and-go" was longer in individuals with delayed recovery (classic $\mathrm{OH}$ or a combination of initial and classic $\mathrm{OH}$ ) as compared to those with initial $\mathrm{OH}$ alone who had a rapid recovery of blood pressure.

The importance of delayed recovery from orthostatic blood pressure changes is illustrated by studies showing that delayed recovery is an independent risk factor for all-cause falls in the upcoming 2 years [7]. Initial $\mathrm{OH}$ on the contrary is not associated with an increased risk for falls. In frail patients, delayed blood pressure recovery and symptoms of orthostatic intolerance occur more frequently $[8,9]$. Furthermore, delayed recovery is associated with increased risk of cognitive impairment, microvascular brain changes, and mortality $[10,11]$.

Another frequently occurring orthostatic phenomenon is delayed $\mathrm{OH}$, defined as a reduction in blood pressure occurring after $3 \mathrm{~min}$ of standing or upright tilt, thus appearing later than classic $\mathrm{OH}$. Delayed $\mathrm{OH}$ is recognized as an earlier phase of classic $\mathrm{OH}$, as $54 \%$ of the individuals with delayed $\mathrm{OH}$ progresses to classic $\mathrm{OH}$ within 10 years [12]. Unfortunately, this was not assessed by Saedon et al., as the time of blood pressure measurements was limited to $3 \mathrm{~min}$. However, delayed $\mathrm{OH}$ is a strong predictor for future frailty: within 10 years $31 \%$ of the individuals with delayed $\mathrm{OH}$ can develop a neurodegenerative synucleinopathy (e.g., Parkinson disease, dementia with Lewy bodies or multiple system atrophy), with increased mortality [12].

In conclusion, initial $\mathrm{OH}$ is a physiological phenomenon in up to $70 \%$ of the elderly. The ability to recover rapidly from initial $\mathrm{OH}$ appears to be a marker for physical fitness. Hence, the true problem is not the presence of initial $\mathrm{OH}$ itself, but the delayed recovery from orthostatic blood pressure changes, which is associated with increased risk of falls, cognitive impairment, and frailty. Like the dilemma of the chicken and the egg, the question is which came first: is frailty causing delayed blood pressure recovery or vice versa? Presumably, both are true. Therefore, future studies should not just focus on initial $\mathrm{OH}$, but also on the factors that could be worsening or improving the delay in blood pressure recovery after standing up, e.g., by exploring the influence of baseline blood pressure, antihypertensive drugs, genetic factors, and training in physical counter-maneuvers.
Funding None.

\section{Compliance with ethical standard}

Conflict of interest The authors declare that they have no conflict of interest.

\section{References}

1. Wieling W, Krediet CT, van Dijk N, Linzer M, Tschakovsky ME (2007) Initial orthostatic hypotension: review of a forgotten condition. Clin Sci (Lond) 112:157-165

2. Cheshire WP Jr (2017) Clinical classification of orthostatic hypotensions. Clin Auton Res 27:133-135

3. van Wijnen VK, Finucane C, Harms MPM, Nolan H, Freeman RL, Westerhof BE, Kenny RA, Ter Maaten JC, Wieling W (2017) Noninvasive beat-to-beat finger arterial pressure monitoring during orthostasis: a comprehensive review of normal and abnormal responses at different ages. J Intern Med 282:468-483

4. Saedon NI, Frith J, Goh CH, Ahmad WAW, Khor HM, Tan KM, Chin AV, Kamaruzzaman SB, Tan MP, Saedah S, Tey NP, Siti Z, Khoo SPL, Rosly HN, Azriyati WNWAA, Ainoriza MA, Chan CS, Wee MC, Por LY, Zaharah H, Norlida A, Firdaus A, Zaherah JS, Rajasuriar R, Sajaratulnish O, Hairi NN, Morgan K, Cumming R, Morris T, MacKenzie L (2019) Orthostatic blood pressure changes and physical, functional and cognitive performance: the MELoR study. Clin Auton Res. https://doi.org/10.1007/s1028 6-019-00647-3 In Press

5. Finucane C, O'Connell MD, Fan CW, Savva GM, Soraghan CJ, Nolan H, Cronin H, Kenny RA (2014) Age-related normative changes in phasic orthostatic blood pressure in a large population study: findings from The Irish Longitudinal Study on Ageing (TILDA). Circulation 130:1780-1789

6. van Twist DJL, Dinh T, Bouwmans EME, Kroon AA (2018) Initial orthostatic hypotension among patients with unexplained syncope: an overlooked diagnosis? Int J Cardiol 271:269-273

7. Finucane C, O'Connell MD, Donoghue O, Richardson K, Savva GM, Kenny RA (2017) Impaired orthostatic blood pressure recovery is associated with unexplained and injurious falls. J Am Geriatr Soc 65:474-482

8. O'Connell MD, Savva GM, Fan CW, Kenny RA (2015) Orthostatic hypotension, orthostatic intolerance and frailty: The Irish Longitudinal Study on Aging-TILDA. Arch Gerontol Geriatr 60:507-513

9. Romero-Ortuno R, Cogan L, O'Shea D, Lawlor BA, Kenny RA (2011) Orthostatic haemodynamics may be impaired in frailty. Age Ageing 40:576-583

10. Hayakawa T, McGarrigle CA, Coen RF, Soraghan CJ, Foran T, Lawlor BA, Kenny RA (2015) Orthostatic blood pressure behavior in people with mild cognitive impairment predicts conversion to dementia. J Am Geriatr Soc 63:1868-1873

11. Kruit MC, Thijs RD, Ferrari MD, Launer LJ, van Buchem MA, van Dijk JG (2013) Syncope and orthostatic intolerance increase risk of brain lesions in migraineurs and controls. Neurology 80:1958-1965

12. Gibbons CH, Freeman R (2015) Clinical implications of delayed orthostatic hypotension: a 10-year follow-up study. Neurology 85:1362-1367 